TRANSACTIONS OF THE

AMERICAN MATHEMATICAL SOCIETY

Volume 355, Number 3, Pages 1201-1212

S 0002-9947(02)03174- 4

Article electronically published on October 25, 2002

\title{
ON THE INVERSION OF THE CONVOLUTION AND LAPLACE TRANSFORM
}

\author{
BORIS BAEUMER
}

\begin{abstract}
We present a new inversion formula for the classical, finite, and asymptotic Laplace transform $\hat{f}$ of continuous or generalized functions $f$. The inversion is given as a limit of a sequence of finite linear combinations of exponential functions whose construction requires only the values of $\hat{f}$ evaluated on a Müntz set of real numbers. The inversion sequence converges in the strongest possible sense. The limit is uniform if $f$ is continuous, it is in $L^{1}$ if $f \in L^{1}$, and converges in an appropriate norm or Fréchet topology for generalized functions $f$. As a corollary we obtain a new constructive inversion procedure for the convolution transform $\mathcal{K}: f \mapsto k \star f$; i.e., for given $g$ and $k$ we construct a sequence of continuous functions $f_{n}$ such that $k \star f_{n} \rightarrow g$.
\end{abstract}

\section{INTRODUCTION}

C. Foiaş [Fo] showed in 1961 that the image of the convolution transform

$$
f \mapsto k \star f:=\int_{0}^{t} k(t-s) f(s) d s
$$

is dense in $L^{1}[0, T]$ for $k, f \in L^{1}[0, T]$ and $0 \in \operatorname{supp}(k)$. This result was later lifted by K. Skórnik $[\mathrm{Sk}]$ to the continuous case. However, the proof is done by contradiction and is not constructive. We will answer the following question: given $k \in L^{1}[0, T]$ with $0 \in \operatorname{supp}(k)$ and $g \in C_{0}([0, T] ; X)$ or $g \in L^{1}([0, T] ; X)$, where $X$ is a Banach space, find a sequence $f_{n} \in C([0, T] ; X)$ such that $k \star f_{n} \rightarrow g$ uniformly, or in the $L^{1}$-norm respectively. The sequence $\left(f_{n}\right)$ is the convolution inverse in the sense of the operational calculus of J. Mikusiński (see [Mi] or Ba]) and converges to a generalized function $f$ in an appropriate norm induced by the function $k$.

We solve this problem by introducing a new inversion formula which can be used for the Laplace transform, the finite Laplace transform and the asymptotic Laplace transform. It is noteworthy that the inversion formula does not involve infinite integrals, infinite sums, derivatives of all orders or the like, but consists of the limit of (finite) linear combinations of exponential functions $\sum_{j=1}^{N} a_{j} e^{\beta_{j} t}$, where the coefficients $a_{j}$ are determined by the (classical, finite, or asymptotic) Laplace transform $\hat{f}$ of $f$, evaluated at Müntz points $\left(\beta_{n}\right)$. This sequence of exponential functions converges uniformly if $f$ is continuous, it converges in $L^{1}$ if $f \in L^{1}$, and it converges in an appropriate norm or Fréchet topology for generalized functions $f$.

Received by the editors January 25, 1999 and, in revised form, August 5, 2002.

2000 Mathematics Subject Classification. Primary 44A35, 44A10, 44A40.

Key words and phrases. Operational calculus, generalized functions, integral transforms. 
We refer to the new inversion formula as the Phragmén-Mikusiński inversion since it generalizes the classical Phragmén-Doetsch inversion of Laplace transform theory (see $[\overline{\mathrm{Do}}$ or $[\mathrm{B}-\mathrm{N}]$ ) and since the proof was inspired by a proof of J. Mikusiński of Titchmarsh's theorem ( $\mathrm{Mi}]$, Chapter VII).

\section{The Phragmén-Mikusiński Inversion}

The first theorem deals with the inversion of the finite Laplace transform. As a corollary we obtain that the inversion formula is indiscriminate towards perturbations of exponential decay which in turn allows the extension to the Laplace transform and to asymptotic Laplace transforms 1

We say that a sequence $\left(\beta_{n}\right) \subset \mathbb{R}^{+}$is a Müntz sequence 2 if for all $n \in \mathbb{N}$,

$$
\beta_{n+1}-\beta_{n} \geq 1 \text { and } \sum_{n=1}^{\infty} \frac{1}{\beta_{n}}=\infty
$$

In the following, let $X$ be an arbitrary complex Banach space.

Theorem 1.1 (Phragmén-Mikusiński inversion on a finite interval). Let $\left(\beta_{n}\right)_{n \in \mathbb{N}}$ be a Müntz sequence and let $N_{n} \in \mathbb{N}$ be such that $\sum_{j=1}^{N_{n}} \frac{1}{\beta_{n j}} \geq T$. Let $f \in C_{0}([0, T] ; X)$ and $q(\lambda):=\int_{0}^{T} e^{-\lambda t} f(t) d t$. Define

$$
\alpha_{n, i}:=\beta_{n i} e^{-\beta_{n i} \sum_{j=1}^{N_{n}} \frac{1}{\beta_{n j}}} \prod_{j=1 ; j \neq i}^{N_{n}} \frac{\beta_{n j}}{\beta_{n j}-\beta_{n i}} .
$$

Then $\left|\alpha_{n, i}\right| \leq \beta_{n i} e^{\frac{1+\ln 2}{n} \beta_{n i}}$ and

$$
f(t)=\lim _{n \rightarrow \infty} \sum_{i=1}^{N_{n}} \alpha_{n, i} q\left(\beta_{n i}\right) e^{\beta_{n i} t},
$$

where the limit is uniform on $[0, S]$ for all $0<S<T$.

Proof. Let $N_{n}$ be such that $c_{n}:=\sum_{j=1}^{N_{n}} \frac{1}{\beta_{n j}} \geq T$. Such $N_{n}$ exists since $\frac{1}{\beta_{n j}}>\frac{1}{\beta_{n j+i}}$ implies that

$$
n \sum_{j=1}^{\infty} \frac{1}{\beta_{n j}} \geq \sum_{i=1}^{n} \sum_{j=1}^{\infty} \frac{1}{\beta_{n j+i}}=\sum_{j=1}^{\infty} \frac{1}{\beta_{j}}-\sum_{j=1}^{n} \frac{1}{\beta_{j}}=\infty .
$$

Thus, $\sum_{j=1}^{\infty} \frac{1}{\beta_{n j}}=\infty$.

The proof of this theorem is built on the fact that the sequence of functions $\phi_{n}: \mathbb{R} \rightarrow \mathbb{R}^{+}$with

$$
\phi_{n}(t):= \begin{cases}\beta_{n} e^{-\beta_{n}(\cdot)} \star \beta_{n 2} e^{-\beta_{n 2}(\cdot)} \star \ldots \star \beta_{n N_{n}} e^{-\beta_{n N_{n}}(\cdot)}\left(t+c_{n}\right) & \text { for } t \geq-c_{n}, \\ 0 & \text { else }\end{cases}
$$

\footnotetext{
${ }^{1}$ It is important to realize that the inversion procedures of Laplace transforms are invariant under certain perturbations. This defines co-classes of functions in the Laplace domain, representing the same function in the time domain.

${ }^{2}$ The condition $\beta_{n+1}-\beta_{n} \geq 1$ can be relaxed to $\beta_{n+1}-\beta_{n} \geq \delta>0$; however, then there exists a subsequence that satisfies the condition.
} 
converges "in the mean" to the $\delta$-function; i.e., the antiderivatives $\Phi_{n}:=1 \star \phi_{n}$ converge towards the Heaviside function $H: t \mapsto \chi_{(0, \infty)}(t)$ (pointwise for all $t \neq 0$ and uniformly for $|t|>\epsilon)$.

(A) We show that $\Phi_{n}(t)=1-\sum_{i=1}^{N_{n}} \frac{\alpha_{n, i}}{\beta_{n i}} e^{-\beta_{n i} t}$ for $t \geq-c_{n}$. Consider $\psi_{n}:=$ $1 \star \beta_{n} e^{-\beta_{n}(\cdot)} \star \ldots \star \beta_{n N_{n}} e^{-\beta_{n N_{n}}(\cdot)}$. Then

$$
\begin{aligned}
\int_{0}^{\infty} e^{-\lambda t} \psi_{n}(t) d t & =\frac{1}{\lambda} \frac{\beta_{n}}{\lambda+\beta_{n}} \cdots \frac{\beta_{n N_{n}}}{\lambda+\beta_{n N_{n}}} \\
& =\gamma_{n, 0} \frac{1}{\lambda}+\gamma_{n, 1} \frac{1}{\lambda+\beta_{n}}+\ldots+\gamma_{n, N_{n}} \frac{1}{\lambda+\beta_{n N_{n}}}
\end{aligned}
$$

where

$$
\gamma_{n, i}=-\prod_{j=1 ; j \neq i}^{N_{n}} \frac{\beta_{n j}}{\beta_{n j}-\beta_{n i}}
$$

Since the inverse Laplace transform of $\frac{1}{\lambda+\beta_{n i}}$ is $e^{-\beta_{n i} t}$, we obtain that $\psi_{n}(t)=$ $1+\sum_{i=1}^{N_{n}} \gamma_{n, i} e^{-\beta_{n i} t}$ for $t \geq 0$. Therefore,

$$
\Phi_{n}(t)=\psi_{n}\left(t+c_{n}\right)=1+\sum_{i=1}^{N_{n}} \gamma_{n, i} e^{-\beta_{n i}\left(t+c_{n}\right)}
$$

for all $t \geq-c_{n}$. Since

$$
\alpha_{n, i}:=\beta_{n i} e^{-\beta_{n i} \sum_{j=1}^{N_{n}} \frac{1}{\beta_{n j}}} \prod_{j=1 ; j \neq i}^{N_{n}} \frac{\beta_{n j}}{\beta_{n j}-\beta_{n i}}=-\beta_{n i} \gamma_{n, i} e^{-\beta_{n i} c_{n}}
$$

we obtain that $\Phi_{n}(t)=1-\sum_{i=1}^{N_{n}} \frac{\alpha_{n, i}}{\beta_{n i}} e^{-\beta_{n i} t}$ for all $t \geq-c_{n}$.

(B) We show that $\left|\alpha_{n, i}\right| \leq \beta_{n i} e^{\frac{1+\ln 2}{n} \beta_{n i}}$. We have that

$$
\ln \left|\frac{\alpha_{n, i}}{\beta_{n i}}\right|=-\beta_{n i} \sum_{j=1}^{N_{n}} \frac{1}{\beta_{n j}}+\sum_{j=1}^{i-1} \ln \frac{\beta_{n j}}{\beta_{n i}-\beta_{n j}}+\sum_{j=i+1}^{N_{n}} \ln \frac{\beta_{n j}}{\beta_{n j}-\beta_{n i}}=: S_{1}+S_{2}+S_{3} .
$$

We first look at $S_{2}$. Since $\beta_{n j} \leq \beta_{n i}-n(i-j)$ for $j<i$, and since the function $t \mapsto \frac{t}{\beta_{n i}-t}$ is increasing on $\left(0, \beta_{n i}\right)$, we know that

$$
\frac{\beta_{n j}}{\beta_{n i}-\beta_{n j}} \leq \frac{\beta_{n i}-n(i-j)}{\beta_{n i}-\left(\beta_{n i}-n(i-j)\right)}=\frac{\beta_{n i}-n(i-j)}{n(i-j)}
$$

and thus

$$
S_{2} \leq \sum_{j=1}^{i-1} \ln \frac{\beta_{n i}-n(i-j)}{n(i-j)}=\sum_{j=1}^{i-1} \ln \frac{\beta_{n i}-n j}{n j}
$$

The fact that the function $t \mapsto \frac{\beta_{n i}-n t}{n t}$ is decreasing for $t>0$ yields

$$
S_{2} \leq \int_{0}^{i-1} \ln \frac{\beta_{n i}-n t}{n t} d t=\frac{\beta_{n i}}{n} \int_{0}^{n(i-1) / \beta_{n i}} \ln \left(\frac{1}{t}-1\right) d t .
$$

Now, $\ln (1 / t-1)>0$ if $t \in(0,1 / 2)$ and $\ln (1 / t-1)<0$ if $t \in(1 / 2,1)$. Thus

$$
S_{2}<\frac{\beta_{n i}}{n} \int_{0}^{1 / 2} \ln \left(\frac{1}{t}-1\right) d t=\frac{\beta_{n i}}{n} \int_{0}^{1 / 2} \frac{1}{1-t} d t=\frac{\beta_{n i} \ln 2}{n} .
$$


In a similar fashion we find an estimate for $S_{1}+S_{3}$. Since the function $t \mapsto$ $-\frac{\beta_{n i}}{t}+\ln \frac{t}{t-\beta_{n i}}$ is positive and decreasing on $\left(\beta_{n i}, \infty\right)$, and since $\beta_{n i}+n(j-i) \leq \beta_{n j}$, we obtain that

$$
\begin{aligned}
S_{1}+S_{3} & =-\sum_{j=1}^{N_{n}} \frac{\beta_{n i}}{\beta_{n j}}+\sum_{j=i+1}^{N_{n}} \ln \frac{\beta_{n j}}{\beta_{n j}-\beta_{n i}}<\sum_{j=i+1}^{\infty}-\frac{\beta_{n i}}{\beta_{n j}}+\ln \frac{\beta_{n j}}{\beta_{n j}-\beta_{n i}} \\
& \leq \sum_{j=i+1}^{\infty}\left(-\frac{\beta_{n i}}{\beta_{n i}+n(j-i)}+\ln \frac{\beta_{n i}+n(j-i)}{n(j-i)}\right) \\
& =\sum_{j=1}^{\infty}\left(-\frac{\beta_{n i}}{\beta_{n i}+n j}+\ln \frac{\beta_{n i}+n j}{n j}\right)<\int_{0}^{\infty}\left(-\frac{\beta_{n i}}{\beta_{n i}+n t}+\ln \frac{\beta_{n i}+n t}{n t}\right) d t \\
& =\frac{\beta_{n i}}{n} \int_{0}^{\infty}\left(-\frac{1}{1+t}+\ln \frac{1+t}{t}\right) d t=\left.\frac{\beta_{n i}}{n}\left(t \ln \frac{1+t}{t}\right)\right|_{t=0} ^{t=\infty}=\frac{\beta_{n i}}{n} .
\end{aligned}
$$

Hence, $\ln \left|\frac{\alpha_{n, i}}{\beta_{n i}}\right|<\frac{\beta_{n i}(1+\ln 2)}{n}$.

(C) We show that $\Phi_{n}(t) \rightarrow 1$ for all $t>0$. Let $t>0$, and let $n$ be such that $\frac{2}{n}<\frac{t}{2}$. Then

$$
\begin{aligned}
\left|\Phi_{n}(t)-1\right| & \leq \sum_{i=1}^{N_{n}}\left|\frac{\alpha_{n, i}}{\beta_{n i}}\right| e^{-\beta_{n i} t} \leq \sum_{i=1}^{\infty} e^{\frac{2 \beta_{n i}}{n}} e^{-\beta_{n i} t} \\
& \leq \sum_{i=1}^{\infty} e^{-\beta_{n i} t / 2} \leq \sum_{i=1}^{\infty} e^{-n i t / 2}=\frac{e^{-n t / 2}}{1-e^{-n t / 2}} .
\end{aligned}
$$

Thus, $\Phi_{n}(t) \rightarrow 1$ as $n \rightarrow \infty$, uniformly for $t>\epsilon>0$.

It follows from the definition of $\phi_{n}$ that $\phi_{n}$, as a convolution of positive functions, is positive. Hence $\Phi_{n}=1 \star \phi_{n}$ is positive and monotonically increasing. Therefore,

$$
\int_{0}^{\infty} e^{-t} \Phi_{n}(t) d t \rightarrow 1
$$

(D) We show that $\int_{-\infty}^{\infty} e^{-t} \Phi_{n}(t) d t \rightarrow 1$, which implies - again by the positivity and monotonicity of $\Phi_{n}-$ that $\Phi_{n}(t) \rightarrow 0$ for all $t<\sqrt{3}$ and thus uniformly for all $t<-\epsilon<0$. Since

$$
\frac{\beta_{n i}+1}{\beta_{n i}}<e^{1 / \beta_{n i}}<\frac{1}{1-1 / \beta_{n i}}=\frac{\beta_{n i}}{\beta_{n i}-1},
$$

we know that

$$
1<\frac{\beta_{n i}}{\beta_{n i}+1} e^{1 / \beta_{n i}}<\frac{\beta_{n i}^{2}}{\beta_{n i}^{2}-1}<1+\frac{1}{(n i)^{2}-1}<e^{1 /\left((n i)^{2}-1\right)} .
$$

By the definition of $\Phi_{n}$,

$$
\begin{aligned}
\int_{-\infty}^{\infty} e^{-t} \Phi_{n}(t) d t & =\int_{-c_{n}}^{\infty} e^{-t} \Phi_{n}(t) d t=e^{c_{n}} \int_{0}^{\infty} e^{-t} \Phi_{n}\left(t-c_{n}\right) d t \\
& =e^{\sum_{i=1}^{N_{n}} 1 / \beta_{n i}} \prod_{i=1}^{N_{n}} \frac{\beta_{n i}}{1+\beta_{n i}}=\prod_{i=1}^{N_{n}} \frac{\beta_{n i}}{1+\beta_{n i}} e^{1 / \beta_{n i}} .
\end{aligned}
$$

\footnotetext{
${ }^{3}$ Suppose there exists $t<0$ such that $\Phi_{n}(t)$ does not converge to 0 . Since $\int_{t}^{0} e^{-s} \Phi_{n}(s) d s \geq$ $\Phi_{n}(t)\left(e^{-t}-1\right)$, it would follow that $\int_{-\infty}^{0} e^{-t} \Phi_{n}(s) d s$ does not converge to 0 either.
} 
Thus,

$$
\begin{aligned}
1 \leq \int_{-\infty}^{\infty} e^{-t} \Phi_{n}(t) d t & =\prod_{i=1}^{N_{n}} \frac{\beta_{n i}}{1+\beta_{n i}} e^{1 / \beta_{n i}} \leq \prod_{i=1}^{\infty} e^{1 /\left((n i)^{2}-1\right)} \\
& =e^{\sum_{i=1}^{\infty} \frac{1}{(n i)^{2}-1}} \rightarrow 1 .
\end{aligned}
$$

(E) Finally, we have the tools necessary to show convergence of the PhragménMikusiński inversion. We know that

$$
\begin{aligned}
& \left\|f(t)-\sum_{i=1}^{N_{n}} \alpha_{n, i} r\left(\beta_{n i}\right) e^{\beta_{n i} t}\right\|=\left\|f(t)-\int_{0}^{T} \sum_{i=1}^{N_{n}} \alpha_{n, i} e^{\beta_{n i} t} e^{-\beta_{n i} s} f(s) d s\right\| \\
& =\left\|f(t)-\int_{0}^{T} \phi_{n}(s-t) f(s) d s\right\| .
\end{aligned}
$$

Let $\epsilon>0$. Choose $\delta>0$ such that $\|f(t)-f(s)\|<\epsilon$ for $|t-s|<2 \delta$, and choose $n_{0}$ such that $\Phi_{n}(-\delta)+1-\Phi_{n}(\delta)<\epsilon$ for all $n>n_{0}$. Then, for $t \in[\delta, T-\delta]$,

$$
\begin{aligned}
\| f(t) & -\int_{0}^{T} \phi_{n}(s-t) f(s) d s\left\|\leq \int_{0}^{t-\delta} \phi_{n}(s-t)\right\| f(s)\left\|d s+\int_{t+\delta}^{T} \phi_{n}(s-t)\right\| f(s) \| d s \\
& +\left\|f(t)-\int_{t-\delta}^{t+\delta} \phi_{n}(s-t) f(t) d s\right\|+\int_{t-\delta}^{t+\delta} \phi_{n}(s-t)\|f(t)-f(s)\| d s \\
\leq & \|f\| \int_{0}^{t-\delta} \phi_{n}(s-t) d s+\|f\| \int_{t+\delta}^{T} \phi_{n}(s-t) d s \\
& +\|f\|\left|1-\int_{t-\delta}^{t+\delta} \phi_{n}(s-t) d s\right|+\epsilon \int_{t-\delta}^{t+\delta} \phi_{n}(s-t) d s \\
\leq & \|f\|\left(\Phi_{n}(-\delta)-\Phi_{n}(-t)\right)+\|f\|\left(\Phi_{n}(T-t)-\Phi_{n}(\delta)\right) \\
& +\|f\|\left|1-\Phi_{n}(\delta)+\Phi_{n}(-\delta)\right|+\epsilon\left(\Phi_{n}(t+\delta)-\Phi_{n}(t-\delta)\right) \leq \epsilon(3\|f\|+1) .
\end{aligned}
$$

For $t \in[0, \delta]$ we have the following estimate (using $f(0)=0$ ):

$$
\begin{aligned}
& \left\|f(t)-\int_{0}^{T} \phi_{n}(s-t) f(s) d s\right\| \\
& \quad \leq\|f(t)\|+\int_{0}^{t+\delta} \phi_{n}(s-t)\|f(s)\| d s+\int_{t+\delta}^{T} \phi_{n}(s-t)\|f(s)\| d s \\
& \quad \leq \epsilon+\epsilon\left(\Phi_{n}(\delta)-\Phi_{n}(-t)\right)+\|f\|\left(\Phi_{n}(T-t)-\Phi_{n}(\delta)\right) \leq \epsilon(2+\|f\|) .
\end{aligned}
$$

Thus $\sum_{i=1}^{N_{n}} \alpha_{n, i} r\left(\beta_{n i}\right) e^{\beta_{n i} t}$ converges uniformly on $[0, S]$ to $f(t)$.

Changing only the final argument of the proof of the previous theorem we obtain convergence in the $L^{1}$-norm if $f \in L^{1}$. In fact, as we will see in Section 2 , if $f$ is in a space of generalized functions, then, modified slightly, the above sum of exponential functions converges in a suitable norm (which is optimal).

Corollary 1.2. Let $\left(\beta_{n}\right)_{n \in \mathbb{N}}, \alpha_{n, i}$, and $N_{n}$ be as in Theorem 1.1, and let $f \in$ $L^{1}([0, T] ; X)$. Then

$$
f=\lim _{n \rightarrow \infty} \sum_{i=1}^{N_{n}} \alpha_{n, i} q\left(\beta_{n i}\right) e^{\beta_{n i}(\cdot)},
$$

where the limit is in the $L^{1}([0, T] ; X)$-norm. 
Proof. By (1.1) we have to find an estimate for $\left\|f(\cdot)-\int_{0}^{T} \phi_{n}(s-\cdot) f(s) d s\right\|_{L^{1}}$. Let $\epsilon>0$. Choose $\delta>0$ such that $\int_{\delta}^{T-\delta}\|f(s+t)-f(s)\| d s<\epsilon$ for $|t|<\delta$. Choose $n_{0}$ such that $\Phi_{n}(-\delta)+1-\Phi_{n}(\delta)<\epsilon$ for all $n>n_{0}$. Extending $f$ by zero we obtain

$$
\begin{aligned}
\| f(\cdot)- & \int_{0}^{T} \phi_{n}(s-\cdot) f(s) d s\left\|_{L^{1}} \leq \int_{0}^{T}\right\| f(t) \|\left(1-\int_{t-\delta}^{t+\delta} \phi_{n}(s-t) d s\right) d t \\
& +\int_{0}^{T} \int_{t-\delta}^{t+\delta} \phi_{n}(s-t)\|f(t)-f(s)\| d s d t \\
& +\int_{0}^{T} \int_{0}^{t-\delta} \phi_{n}(s-t)\|f(s)\| d s d t+\int_{0}^{T} \int_{t+\delta}^{T} \phi_{n}(s-t)\|f(s)\| d s d t \\
\leq & \|f\|_{L^{1}}\left(1-\Phi_{n}(\delta)+\Phi_{n}(-\delta)\right)+\int_{-\delta}^{\delta} \int_{0}^{T} \phi_{n}(s)\|f(t)-f(s+t)\| d t d s \\
& +\int_{0}^{T-\delta} \int_{s+\delta}^{T} \phi_{n}(s-t)\|f(s)\| d t d s+\int_{\delta}^{T} \int_{0}^{s-\delta} \phi_{n}(s-t)\|f(s)\| d t d s \\
\leq & \epsilon(\|f\|+1)+\int_{0}^{T-\delta}\|f(s)\|\left(\Phi_{n}(-\delta)-\Phi_{n}(s-T)\right) d s \\
& +\int_{\delta}^{T}\|f(s)\|\left(\Phi_{n}(s)-\Phi_{n}(\delta)\right) d s \\
\leq & \epsilon(3\|f\|+1) .
\end{aligned}
$$

In order to extend the inversion formula to Laplace or asymptotic Laplace transforms of functions defined on $[0, \infty)$, we need the notion of exponential decay. We say a function is of exponential decay $T>0$ if

$$
\limsup _{\lambda \rightarrow \infty} \frac{1}{\lambda} \ln \|r(\lambda)\| \leq-T \text {. }
$$

This notion is important since, as we see next, the Phragmén-Mikusiński inversion does not register perturbations of exponential decay $T$; i.e., if for $t \in[0, T)$,

$$
f(t)=\lim _{n \rightarrow \infty} \sum_{i=1}^{N_{n}} \alpha_{n, i} q\left(\beta_{n i}\right) e^{\beta_{n i} t}
$$

for some function $q$, then

$$
f(t)=\lim _{n \rightarrow \infty} \sum_{i=1}^{N_{n}} \alpha_{n, i} \tilde{q}\left(\beta_{n i}\right) e^{\beta_{n i} t}
$$

for all perturbed functions $\tilde{q}=q+r$, where $r$ is some perturbation of exponential decay $T$.

Corollary 1.3. Let $r:(\omega, \infty) \rightarrow X$ be a function, and let $\left(\beta_{n}\right)_{n \in \mathbb{N}} \subset \mathbb{R}^{+}$be a Müntz sequence. If $r$ is of exponential decay $T>0$, then

$$
\lim _{n \rightarrow \infty} \sum_{i=1}^{N_{n}} \alpha_{n, i} e^{\beta_{n i} t} r\left(\beta_{n i}\right)=0
$$

for all $0 \leq t<T$, where $\alpha_{n, i}$ and $N_{n}$ are as in the previous theorem, and the limit is uniform for all $t \in[0, S]$ and $0<S<T$. 
Proof. Let $t \in[0, T)$. Then $-T<-\frac{2 T+t}{3}$. Thus, there exists $n_{0}$ such that $\left\|r\left(\beta_{n}\right)\right\| \leq \frac{1}{\beta_{n}} e^{-\frac{2 T+t}{3} \beta_{n}}$ for all $n \geq n_{0}$ and such that $2 / n_{0}<(T-t) / 3$. By the previous theorem we know that $\left|\alpha_{n, i}\right|<\beta_{n i} e^{\frac{2}{n} \beta_{n i}}<\beta_{n i} e^{\frac{T-t}{3} \beta_{n i}}$. Thus

$$
\begin{aligned}
\left\|\sum_{i=1}^{N_{n}} \alpha_{n, i} e^{\beta_{n i} t} r\left(\beta_{n i}\right)\right\| & \leq \sum_{i=1}^{\infty} e^{\frac{T+2 t}{3} \beta_{n i}} e^{-\frac{2 T+t}{3} \beta_{n i}}=\sum_{i=1}^{\infty} e^{-\frac{T-t}{3} \beta_{n i}} \\
& \leq \sum_{i=1}^{\infty} e^{-\frac{T-t}{3} n i}=\frac{e^{-\frac{T-t}{3} n}}{1-e^{-\frac{T-t}{3} n}} \rightarrow 0
\end{aligned}
$$

as $n \rightarrow \infty$, uniformly for all $t \in[0, S]$ for all $0<S<T$.

Asymptotic Laplace transforms appear as an extension of the usual Laplace transform to map functions which are not exponentially bounded and were first introduced by J.C. Vignaux in 1939 (see [Vi] or [L-N]). The asymptotic Laplace transform of a function $f \in L_{l o c}^{1}([0, \infty) ; X)$ is defined to be an equivalence class of analytic functions defined in a post sectorial region $\Omega$ of the complex plane with the following property:

$$
\mathcal{L}_{A}(f)=\left\{r \in \mathcal{A}(\Omega, X): r \approx_{T} \lambda \mapsto \int_{0}^{T} e^{-\lambda t} f(s) d s \text { for all } T>0\right\}
$$

where $r \approx_{T} q$ if $r-q$ is of exponential decay $T$. This set is always nonempty and the properties of the Laplace transform extend fully (see [L-N] or [Ba]). With the help of Corollaries 1.2 and 1.3 we obtain the following result.

Corollary 1.4. Let $f \in C_{0}([0, \infty) ; X)$ or $f \in L_{\text {loc }}^{1}([0, \infty) ; X)$ and $\hat{f}(\lambda) \in \mathcal{L}_{A}(f)$. Let $\left(\beta_{n}\right)_{n \in \mathbb{N}}$ be a Müntz sequence. Let $N_{n}$ be such that $\sum_{i=1}^{N_{n}} \frac{1}{\beta_{n i}} \rightarrow \infty$ as $n \rightarrow \infty$, and let $\alpha_{n, i}$ be as in Theorem 1.1. Then, for all $t \geq 0$,

$$
f=\lim _{n \rightarrow \infty} \sum_{i=1}^{N_{n}} \alpha_{n, i} \hat{f}\left(\beta_{n i}\right) e^{\beta_{n i}(\cdot)}
$$

where the limit is uniform on compact sets or taken in $L_{\text {loc }}^{1}$, respectively.

Proof. Let $0<S<T, q(\lambda):=\int_{0}^{T} e^{-\lambda t} f(t) d t$, and $r(\lambda):=\hat{f}(\lambda)-q(\lambda)$. Then $\hat{f}=q+r$ and

$$
\sum_{n=1}^{N_{k}} \alpha_{k, n} e^{t \beta_{k n}} \hat{f}\left(\beta_{k n}\right)=\sum_{n=1}^{N_{k}} \alpha_{k, n} e^{t \beta_{k n}} q\left(\beta_{k n}\right)+\sum_{n=1}^{N_{k}} \alpha_{k, n} e^{t \beta_{k n}} r\left(\beta_{k n}\right) .
$$

By Theorem 1.1 for $f \in C_{0}([0, \infty) ; X)$, the first term converges uniformly on $[0, S]$ to $f(t)$. By Corollary 1.2 , the first term converges in $L^{1}$ for $f \in L_{l o c}^{1}([0, \infty) ; X)$. By Corollary 1.3, the second term converges uniformly to 0 on $[0, S]$.

An immediate consequence is the inversion formula for the Laplace transform.

Corollary 1.5. Let $f \in C_{0}([0, \infty) ; X)$ or $f \in L_{l o c}^{1}([0, \infty) ; X)$ be a Laplace transformable function and let $\hat{f}(\lambda)=\int_{0}^{\infty} e^{-\lambda t} f(t) d t$ for sufficiently large $\operatorname{Re}(\lambda)$. Let $\left(\beta_{n}\right)_{n \in \mathbb{N}}$ be a Müntz sequence. Let $N_{n}$ be such that $\sum_{i=1}^{N_{n}} \frac{1}{\beta_{n i}} \rightarrow \infty$ as $n \rightarrow \infty$, and let $\alpha_{n, i}$ be as in Theorem 1.1. Then, for all $t \geq 0$,

$$
f=\lim _{n \rightarrow \infty} \sum_{i=1}^{N_{n}} \alpha_{n, i} \hat{f}\left(\beta_{n i}\right) e^{\beta_{n i}(\cdot)}
$$


where the limit is uniform on compact sets or taken in $L_{l o c}^{1}$, respectively.

Another consequence is the following statement characterizing the maximal interval $[0, T]$ on which a function can vanish in terms of the growth of its asymptotic Laplace transform evaluated at Müntz points. It will furthermore allow us to avoid singularities that might appear in the Laplace transform of generalized functions (see below).

Theorem 1.6. Let $0 \leq T$ and let $f \in L_{l o c}^{1}([0, \infty) ; X)$ with $\hat{f} \in \mathcal{L}_{A}(f)$. Then the following are equivalent 4

(i) Every Müntz sequence $\left(\beta_{n}\right)_{n \in \mathbb{N}}$ satisfies

$$
\limsup _{n \rightarrow \infty} \frac{1}{\beta_{n}} \ln \left\|\hat{f}\left(\beta_{n}\right)\right\|=-T .
$$

(ii) For every Müntz sequence $\left(\beta_{n}\right)_{n \in \mathbb{N}}$ there exists a Müntz subsequence $\left(\beta_{n_{k}}\right)_{k \in \mathbb{N}}$ satisfying

$$
\lim _{k \rightarrow \infty} \frac{1}{\beta_{n_{k}}} \ln \left\|\hat{f}\left(\beta_{n_{k}}\right)\right\|=-T .
$$

(iii) There exists a Müntz sequence $\left(\beta_{n}\right)_{n \in \mathbb{N}}$ satisfying

$$
\limsup _{n \rightarrow \infty} \frac{1}{\beta_{n}} \ln \left\|\hat{f}\left(\beta_{n}\right)\right\|=-T .
$$

(iv) $f(t)=0$ almost everywhere on $[0, T]$ and $T \in \operatorname{supp}(f)$.

(v) $\lim \sup _{\lambda \rightarrow \infty} \frac{1}{\lambda} \ln \|\hat{f}(\lambda)\|=-T$.

Proof. We show first that $(i) \Rightarrow(i i) \Rightarrow($ iii $) \Rightarrow(i v) \Rightarrow(i)$ and then $(i v) \Leftrightarrow$ $(v)$. Suppose $(i)$ holds, and let $\left(\beta_{n}\right)_{n \in \mathbb{N}}$ be a Müntz sequence. Let $\left(\beta_{n}^{\epsilon}\right)_{n \in \mathbb{N}}$ be the subsequence that is obtained by dropping the elements of $\left(\beta_{n}\right)_{n \in \mathbb{N}}$ for which $\left\|r\left(\beta_{n}\right)\right\| \leq e^{(-T-\epsilon) \beta_{n}}$. The dropped subsequence $\left(\gamma_{n}^{\epsilon}\right)$ satisfies

$$
\limsup _{n \rightarrow \infty} \frac{1}{\gamma_{n}^{\epsilon}} \ln \left\|\hat{f}\left(\gamma_{n}^{\epsilon}\right)\right\| \leq-T-\epsilon .
$$

Since $(i)$ is assumed to hold, the dropped sequence $\left(\gamma_{n}^{\epsilon}\right)$ cannot satisfy the Müntz condition. Thus $\sum \frac{1}{\gamma_{n}^{\epsilon}}<\infty$, and hence $\left(\beta_{n}^{\epsilon}\right)_{n \in \mathbb{N}}$ is still a Müntz sequence. Now we use a diagonal argument. Let $j=1$ and take the first $k_{1}$ elements of $\left(\beta_{n}^{1}\right)_{n \in \mathbb{N}}$ such that $\sum_{i=1}^{k_{1}} \frac{1}{\beta_{i}^{\mathbf{1}}}>1$. Continue with elements of the sequence $\left(\beta_{n}^{1 / 2}\right)$, picking consecutive elements until $\sum_{j=1}^{k_{1}} \frac{1}{\beta_{j}^{1}}+\sum_{j=k_{1}}^{k_{2}} \frac{1}{\beta_{j}^{1 / 2}} \geq 2$. Continuing this process we end up with a subsequence having the properties stated in $(i i)$.

Clearly (ii) implies (iii). Suppose (iii) holds. In the case that $T>0$, we combine Corollary 1.2 with Corollary 1.3 and obtain that $\int_{0}^{t} f(s) d s=0$ for all $t \in[0, T)$. Now let $T \geq 0$ and suppose that $f=0$ almost everywhere on $[0, T+\epsilon]$. However, by the definition of asymptotic Laplace transforms, there exists a remainder function $r \approx_{T+\epsilon} 0$ such that $\hat{f}(\lambda)=\int_{0}^{T+\epsilon} e^{-\lambda t} f(t) d t+r(\lambda)=r(\lambda)$, contradicting (iii). Thus (iv) holds.

\footnotetext{
${ }^{4}$ G. Doetsch ([Do], Satz 14.3.1) proved that $\lim \sup { }_{\lambda \rightarrow \infty} \frac{1}{\lambda} \ln \|\hat{f}(\lambda)\| \leq-T$ is equivalent to the statement $(i v)^{\prime}: f=0$ on $[0, T]$ a.e. In fact, it follows from the proof below that statement $(i v)^{\prime}$ is equivalent to the statements $(i)-(i i i)$ if $\lim _{\sup } \rightarrow \infty \frac{1}{\beta_{n}} \ln \left\|\hat{f}\left(\beta_{n}\right)\right\|=-T$ is replaced by $\lim \sup _{n \rightarrow \infty} \frac{1}{\beta_{n}} \ln \left\|\hat{f}\left(\beta_{n}\right)\right\| \leq-T$.
} 
Suppose (iv) holds. Then $\lim \sup _{\lambda \rightarrow \infty} \frac{1}{\lambda} \ln \|\hat{f}(\lambda)\| \leq-T$. Suppose

$$
\limsup _{n \rightarrow \infty} \frac{1}{\beta_{n}} \ln \left\|\hat{f}\left(\beta_{n}\right)\right\| \leq-T-\epsilon .
$$

Then Corollary 1.2 and Corollary 1.3 imply that $\int_{0}^{t} f(s) d s=0$ for $t \in[0, T+\epsilon)$, contradicting $T \in \operatorname{supp}(f)$. Thus $(i)$ holds. Furthermore, suppose

$$
\limsup _{\lambda \rightarrow \infty} \frac{1}{\lambda} \ln \|\hat{f}(\lambda)\|<-T \text {. }
$$

This would contradict $(i)$ and therefore $(v)$ has to hold.

Suppose $(v)$ holds. Thus $f$ vanishes on $[0, T]$. Suppose $f$ vanishes on $[0, T+\epsilon]$. Then $\lim \sup _{\lambda \rightarrow \infty} \frac{1}{\lambda} \ln \|\hat{f}(\lambda)\| \leq-T-\epsilon$, contradicting $(v)$. Thus $(i v)$ holds.

As a corollary we obtain a short and elegant proof of Titchmarsh's theorem, following an idea of J. Mikusiński for the scalar-valued case [Mi].

Corollary 1.7 (E. C. Titchmarsh's theorem). Let $0<T$, and let $k \in L_{\text {loc }}^{1}[0, \infty)$ and $f \in L_{l o c}^{1}([0, \infty) ; X)$. Then $k \star f=0$ on $[0, T]$ implies that there exist $x_{1}, x_{2} \geq 0$ with $x_{1}+x_{2} \geq T$ s.t. $k=0$ a.e. on $\left[0, x_{1}\right]$ and $f=0$ a.e. on $\left[0, x_{2}\right]$.

Proof. Let $\hat{k} \in \mathcal{L}_{A}(k)$ and $\hat{f} \in \mathcal{L}_{A}(f)$. Let $x_{1}:=\lim \sup _{\lambda \rightarrow \infty} \frac{1}{\lambda} \ln |\hat{k}(\lambda)|$ and $x_{2}:=$ $\lim \sup _{\lambda \rightarrow \infty} \frac{1}{\lambda} \ln \|\hat{f}(\lambda)\|$. By Theorem[1.6, $k$ and $f$ are 0 a.e. on $\left[0, x_{1}\right]$ and on $\left[0, x_{2}\right]$ respectively. Furthermore, by taking subsequences, there exists a Müntz sequence $\left(\beta_{n}\right)_{n \in \mathbb{N}}$ such that $x_{1}=-\lim _{n \rightarrow \infty} \frac{1}{\beta_{n}} \ln \left|\hat{k}\left(\beta_{n}\right)\right|$ and $x_{2}=-\lim _{n \rightarrow \infty} \frac{1}{\beta_{n}} \ln \left\|\hat{f}\left(\beta_{n}\right)\right\|$. Since

$$
\lim _{n \rightarrow \infty} \frac{1}{\beta_{n}} \ln \left\|\hat{k}\left(\beta_{n}\right) \hat{f}\left(\beta_{n}\right)\right\|=\lim _{n \rightarrow \infty} \frac{1}{\beta_{n}} \ln \left|\hat{k}\left(\beta_{n}\right)\right|+\lim _{n \rightarrow \infty} \frac{1}{\beta_{n}} \ln \left\|\hat{f}\left(\beta_{n}\right)\right\|=-x_{1}-x_{2},
$$

we obtain that $x_{1}+x_{2} \geq T$.

\section{The Inversion of the Convolution Transform}

J. Mikusiński introduced generalized functions as elements in the quotient field of continuous functions with convolution as multiplication. Into this purely algebraic approach he inserted a topology. A sequence of generalized functions $\frac{f_{n}}{g_{n}}$ is said to converge to $\frac{f}{g}$ if there exists a $k \in C[0, \infty)$ such that $k \star \frac{f_{n}}{g_{n}} \in C[0, \infty)$ for all $n$ and $k \star \frac{f_{n}}{g_{n}} \rightarrow k \star \frac{f}{g}$ (see [Mi] for details).

We use this topology to introduce Banach spaces (Fréchet spaces, Fréchet lattices, etc.) of generalized functions. We define generalized functions $g:[0, \infty) \rightarrow X$ as elements of the completion $C^{[k]}([0, \infty) ; X)$ of the vector space $C([0, \infty) ; X)$ equipped with seminorms

$$
\|f\|_{n}:=\|k \star f\|_{n}=\sup _{t \in[0, n}\left\|\int_{0}^{t} k(t-s) f(s) d s\right\|,
$$

where $k \in L_{l o c}^{1}[0, \infty]$ with $0 \in \operatorname{supp}(k)$ (see [Ba or $[\mathrm{B}-\mathrm{L}-\mathrm{N}]$ ). Similarly, we define the spaces $L^{1,[k]}([0, \infty) ; X)$ as completions, measuring a function by the $L^{1}$-norm of its convolution image. Hereby, the following theorem is crucial (see also [Fo, [Ti], or [B-L-N]).

Theorem 2.1 (Titchmarsh-Foias). Let $k \in L^{1}[0, T]$ and consider the convolution $\mathcal{K}: f \mapsto k \star f$ as an operator from $C([0, T] ; X)$ into $C_{0}([0, T] ; X)$ or as an element of $\mathcal{L}\left(L^{1}([0, T] ; X)\right)$. Then the following are equivalent: 
(i) $0 \in \operatorname{supp}(k)$;

(ii) $\mathcal{K}$ is injective;

(iii) the range of $\mathcal{K}$ is dense.

Moreover, $\mathcal{K}$ then extends to an isometric isomorphism between $C^{[k]}([0, T] ; X)$ and $C_{0}([0, T] ; X)$ as well as between $L^{1,[k]}([0, T] ; X)$ and $L^{1}([0, T] ; X)$.

Notice that in Corollary 1.7 we proved the equivalence of $(i)$ and $(i i)$. In Theorem 2.2 we will not only prove the equivalence of $(i)$ and $($ iii $)$, but give an approximating sequence and thus provide an inversion for the convolution transform.

With the above definition, a generalized function $g$ can be regarded as an equivalence class $\left[g_{n}\right]_{k}$ of continuous (or $L^{1}$ ) functions $g_{n}$ such that $k \star g_{n} \rightarrow f=: k \star g$. The union of these spaces will, for the scalar-valued case and up to translation, give us back the convolution field. In the vector-valued case we obtain a vector space over the convolution field (see $\mathrm{Ba}$ for details). Since the (asymptotic) Laplace transform maps convolution into multiplication, the (asymptotic) Laplace transform of such a generalized function is a quotient of the (asymptotic) Laplace transform of a function $f$ and the (asymptotic) Laplace transform of the kernel $k$; thus they are meromorphic functions in some sectorial region in the right half-plane (see $\mathrm{Ba}$. or L-N]).

In the following theorem, for given $k$ and $f$, we provide a sequence of continuous functions $g_{n}$ such that $k \star g_{n} \rightarrow f$. This is done by using the inversion formula on $\hat{g}=\frac{\hat{f}}{\hat{k}}$, yielding the sequence of exponential functions $g_{n}$. This sequence converges in the $\|\cdot\|_{k}$-norm to the generalized function $g$. Hence the inversion formula of Corollary 1.4 not only converges uniformly for continuous functions $f$ or in the $L^{1}$ norm for Bochner integrable $f$, but, in a slightly extended version, it also converges in the $C^{[k]}$-norm or the $L^{1,[k]}$-norm for generalized functions $g \in C^{[k]}([0, \infty) ; X)$ or $g \in L^{1,[k]}([0, \infty) ; X)$.

Theorem 2.2 (Inversion of the convolution transform). Let $k \in L_{\text {loc }}^{1}[0, \infty)$ with $0 \in \operatorname{supp}(k)$ and $\hat{k} \in \mathcal{L}_{A}(k)$. Let $f \in C_{0}([0, \infty) ; X)$ or $f \in L_{l o c}^{1}([0, \infty) ; X)$ and $\hat{f} \in \mathcal{L}_{A}(f)$. Let $\beta_{n}$ be a Müntz sequenc可 such that $\lim _{n \rightarrow \infty} \frac{1}{\beta_{n}} \ln \left\|\hat{k}\left(\beta_{n}\right)\right\|=0$. Let $\epsilon_{n}=\max \left\{\frac{2}{\sqrt{n}}, \frac{-2}{\beta_{n}} \ln \left\|\frac{\hat{k}\left(\beta_{n}\right)}{\beta_{n}}\right\|\right\}$ and

$$
g_{n}(t):=\sum_{i=1}^{N_{n}} \alpha_{n, i} \frac{\hat{f}\left(\beta_{n i}\right)}{\hat{k}\left(\beta_{n i}\right)} e^{\beta_{n i}\left(t-\epsilon_{n}\right)},
$$

where $\alpha_{n, i}$ and $N_{n}$ are defined as in Corollary 1.4. Then

$$
k \star g_{n} \rightarrow f,
$$

where the limit is uniform on compact intervals or taken in $L_{l o c}^{1}$, respectively.

Proof. Let $0<S<T$. Let $r_{T}$ be the remainder function such that

$$
\hat{k}(\lambda)+r_{T}(\lambda)=\int_{0}^{T} e^{-\lambda t} k(s) d s
$$

\footnotetext{
${ }^{5}$ By Theorem [1.6 such a sequence can always be constructed.
} 
for all $\lambda>0$. Then, for $0 \leq t \leq S$,

$$
\begin{aligned}
\left(k \star g_{n}\right)(t)= & \sum_{i=1}^{N_{n}} \alpha_{n, i} \frac{\hat{f}\left(\beta_{n i}\right)}{\hat{k}\left(\beta_{n i}\right)} \int_{0}^{t} k(s) e^{\beta_{n i}\left(t-\epsilon_{n}-s\right)} d s \\
= & \sum_{i=1}^{N_{n}} \alpha_{n, i} \frac{\hat{f}\left(\beta_{n i}\right)}{\hat{k}\left(\beta_{n i}\right)} e^{\beta_{n i}\left(t-\epsilon_{n}\right)} \int_{0}^{t} e^{-\beta_{n i s}} k(s) d s \\
= & \sum_{i=1}^{N_{n}} \alpha_{n, i} \frac{\hat{f}\left(\beta_{n i}\right)}{\hat{k}\left(\beta_{n i}\right)} e^{\beta_{n i}\left(t-\epsilon_{n}\right)} \hat{k}\left(\beta_{n i}\right) \\
& +\sum_{i=1}^{N_{n}} \alpha_{n, i} \frac{\hat{f}\left(\beta_{n i}\right)}{\hat{k}\left(\beta_{n i}\right)} e^{\beta_{n i}\left(t-\epsilon_{n}\right)}\left(r_{T}\left(\beta_{n i}\right)-\int_{t}^{T} e^{-\beta_{n i} s} k(s) d s\right) .
\end{aligned}
$$

By the Phragmén-Mikusiński inversion, the first term converges uniformly (or in $L^{1}$, respectively) to $f$.

We conclude the proof by showing that the second term converges to zero. By the definition of $\epsilon_{n}$ we know that $\left|\hat{k}\left(\beta_{n i}\right)\right| \geq \beta_{n i} e^{-\beta_{n i} \epsilon_{n} / 2}$. Thus, there exists a constant $C$ such that, for $n \rightarrow \infty$,

$$
\begin{aligned}
& \left\|\sum_{i=1}^{N_{n}} \alpha_{n, i} \frac{\hat{f}\left(\beta_{n i}\right)}{\hat{k}\left(\beta_{n i}\right)} e^{\beta_{n i}\left(t-\epsilon_{n}\right)}\left(r_{T}\left(\beta_{n i}\right)-\int_{t}^{T} e^{-\beta_{n i} s} k(s) d s\right)\right\| \\
& \quad \leq \sum_{i=1}^{\infty} \beta_{n i} e^{2 \beta_{n i} / n} \frac{C}{\beta_{n i} e^{-\beta_{n i} \epsilon_{n} / 2}} e^{\beta_{n i}\left(t-\epsilon_{n}\right)} e^{-\beta_{n i} t}=C \sum_{i=1}^{\infty} e^{\beta_{n i}\left(2 / n-\epsilon_{n} / 2\right)} \rightarrow 0 .
\end{aligned}
$$

Remark 2.3. We only considered scalar-valued kernels $k$ in this article. If the kernel is a strongly continuous or strongly locally integrable operator family $\left(K_{t}\right)_{t \geq 0}$, the same theorems hold, provided that for $\hat{K} \in \mathcal{L}_{A}(K)$, there exists a Müntz sequence $\left(\beta_{n}\right)_{n \in \mathbb{N}}$ such that for all $\epsilon>0$ there exists a constant $N_{\epsilon}$ with

$$
\left\|\hat{K}\left(\beta_{n}\right)\right\|\|x\| \leq e^{\beta_{n} \epsilon}\left\|\hat{K}\left(\beta_{n}\right) x\right\|
$$

for all $n>N_{\epsilon}$ and all $x \in X$ (see $\mathrm{Ba}$ for details).

\section{ACKNOWLEDGMENTS}

I am grateful for the help and many valuable suggestions of my advisor, Professor F. Neubrander, at Louisiana State University.

\section{REFERENCES}

[Ba] Baeumer, B. A Vector-Valued Operational Calculus and Abstract Cauchy Problems. Dissertation, Louisiana State University, 1997. (http://math.lsu.edu/ ${ }^{\sim}$ tiger )

[B-L-N] Bäumer, B., G. Lumer, and F. Neubrander. Convolution kernels and generalized functions. Generalized functions, operator theory, and dynamical systems (Brussels, 1997), 68-78, Res. Notes Math. 399, Chapman \& Hall/CRC, Boca Raton, FL, 1999. MR 2000a:44004

[B-N] Bäumer, B. and F. Neubrander. Laplace transform methods for evolution equations. Conferenze del Seminario di Matematica dell'Universitá di Bari, 259, 27-60, 1994. MR 97e:47119

[Do] Doetsch, G. Handbuch der Laplace Transformation. Vol. I-III, Birkhäuser Verlag, BaselStuttgart, 1950-1956. MR 13:230f MR 18:35a MR 18:894c 
[Fo] Foiaş, C. Approximation des opérateurs de J. Mikusiński par des fonctions continues. Studia Mathematica 21, 73-74, 1961. MR 25:3334

[L-N] Lumer, G. and F. Neubrander. Asymptotic Laplace transforms and evolution equations. Evolution equations, Feshbach resonances, singular Hodge theory, 37-57, Math. Top. 16, Wiley-VCH, Berlin, 1999. MR 2000f:47068

[Mi] Mikusiński, J. Operational Calculus. v. 1-2, Pergamon Press, 2nd edition, 1987. MR 86b:44017 MR 88k:44010

[Sk] Skórnik, K. On the Foiaş theorem on convolution of continuous functions. Complex Analysis and Applications '85 (Varna, 1985), 604-608, Publ. House Bulgar. Acad. Sci., Sofia, 1986. MR 89j:46057

[Ti] Titchmarsh, E. C. The zeros of certain integral functions. Proceedings of the London Mathematical Society 25 (1926), 283-302.

[Vi] Vignaux, J. C. Sugli integrali di Laplace asintotici, Atti Accad. naz. Lincei, Rend. Cl. Sci. fis. mat. (6) 29 (1939), 345-400.

Department of Mathematics and Statistics, University of Otago, P.O. Box 56, Dunedin, New Zealand

E-mail address: bbaeumer@maths.otago.ac.nz 